\title{
Osteoporosis and osteopenia in older Emirati men with type 2 diabetes mellitus
}

\author{
R A Almazrouei, F M Alkaabi, H El Gayar, N Lessan, M T Barakat \\ Imperial College London Diabetes Centre, Abu Dhabi, UAE
}

\section{Introduction}

- Osteoporosis is an important cause of morbidity and mortality in older men.

- The extent of this problem amongst Emiratis men has not been previously reported.

- Men constituted only $8.8 \%$ of all the DEXA referrals in a single center study in UAE which showed osteoporosis in $22.5 \%$ and osteopenia in $49 \%$ among 7 I screened men (mixed population) with mean age $55.2(\mathrm{I})$.

- In another local study to assess osteoporosis prevalence, men constituted only I3\% of screened individuals (2)

\section{Aim}

- To establish the prevalence of osteoporosis/ osteopenia among elderly men with type 2 diabetes.

- To investigate the associated risk factors with osteoporosis/osteopenia in elderly men.

\section{Methods}

- A screening program with DEXA for men over age 70 has been implemented in a large diabetes center in the UAE.

- T-score at lumbar spine, femoral necks and hips were retrieved from DEXA scan reports for 179 men with type 2 diabetes over age 70 .

- Relevant demographic, phenotypic data and laboratory investigations at or closest to the time of DEXA and comorbidities list were extracted from individual computerized patient records.

- Stata I 3.0 was used for data analysis.

Figure I: Prevelance of Diffrent Bone Mineral Density in Older Emirati men with Type 2 Diabetes Mellitus

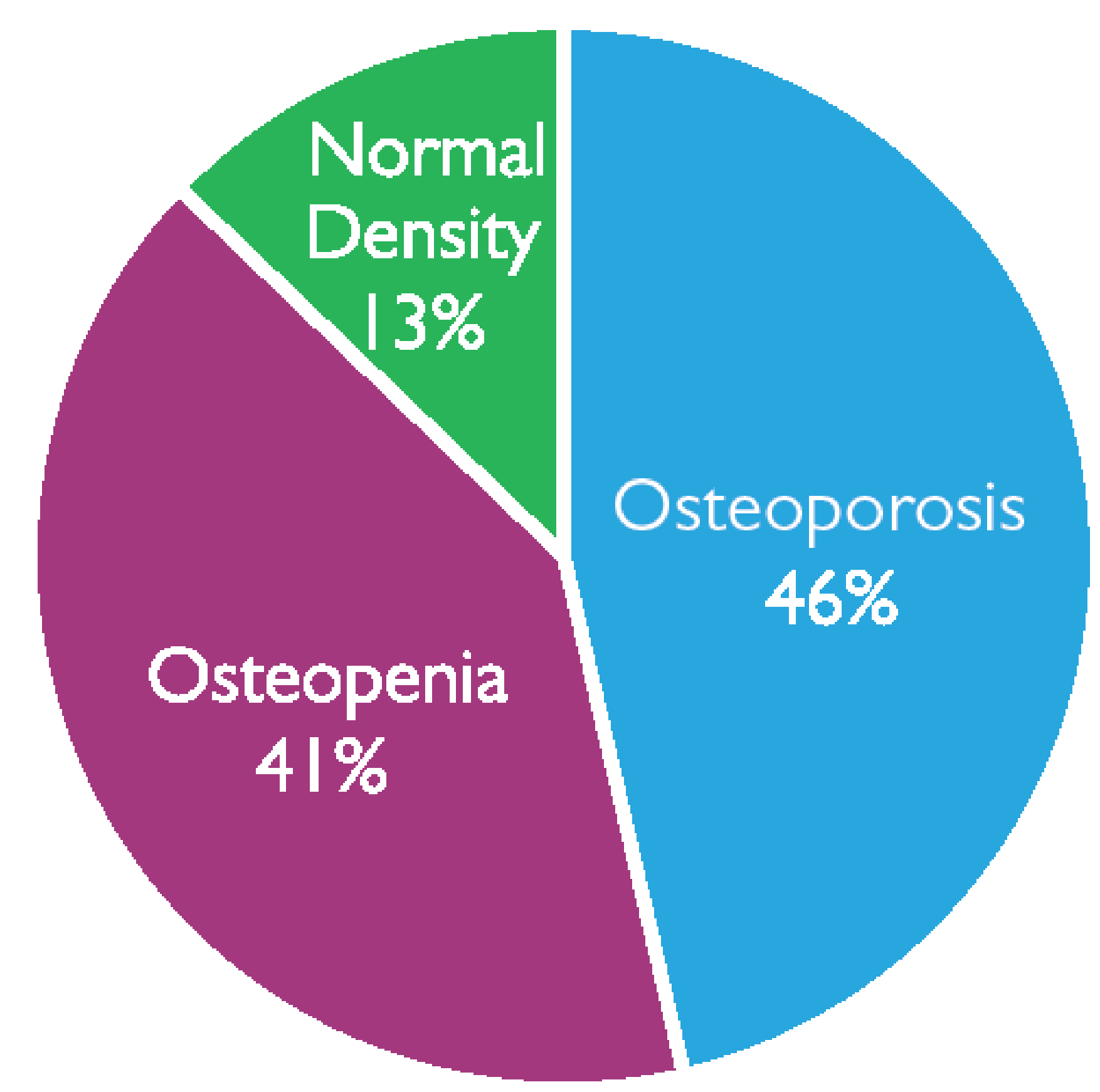

- Osteoporosis - Osteopenia - Normal Density

\section{Results}

- DEXA screening showed osteoporosis in $46 \%$ and osteopenia in $41 \%$ of screened men based on WHO criteria (figure I).

- Significant differences $(p<0.00 \mathrm{I})$ in weight and height were observed between different categories of bone mineral density status (table I).

- Smoking status, comorbidities (cardiovascular disease, hypertension, hypothyroidism), TSH level, DM duration and DM therapy (insulin and OHAs) were not significantly different between different BMD categories.

- $86 \%$ and $22 \%$ of the screened men were on vitamin D and calcium supplements respectively (table I).

- Based only on lumbar spine T-score, $32.2 \%$ have osteoporosis compared to $19.5 \%$ in left femoral neck, $25.4 \%$ in right femoral neck, $5.2 \%$ in left hip and 5.8\% in right hip (figure 2).

- In univariate regression analysis, significant predictors of bone density included age (OR I.|4, $p=0.02)$, weight $(O R$ 0.96, $p=0.02)$ and height $(O R$ 0.87, $p<0.00 I)$. In multivariate analysis including age, weight, height and eGFR, only height (OR 0.88, $p=0.005)$ remained a predictor with significance.

- There was $82.4 \%$ osteoporosis range T-scores concordance between the right \& left femoral neck levels and $88.9 \%$ concordance between right \& left hip.

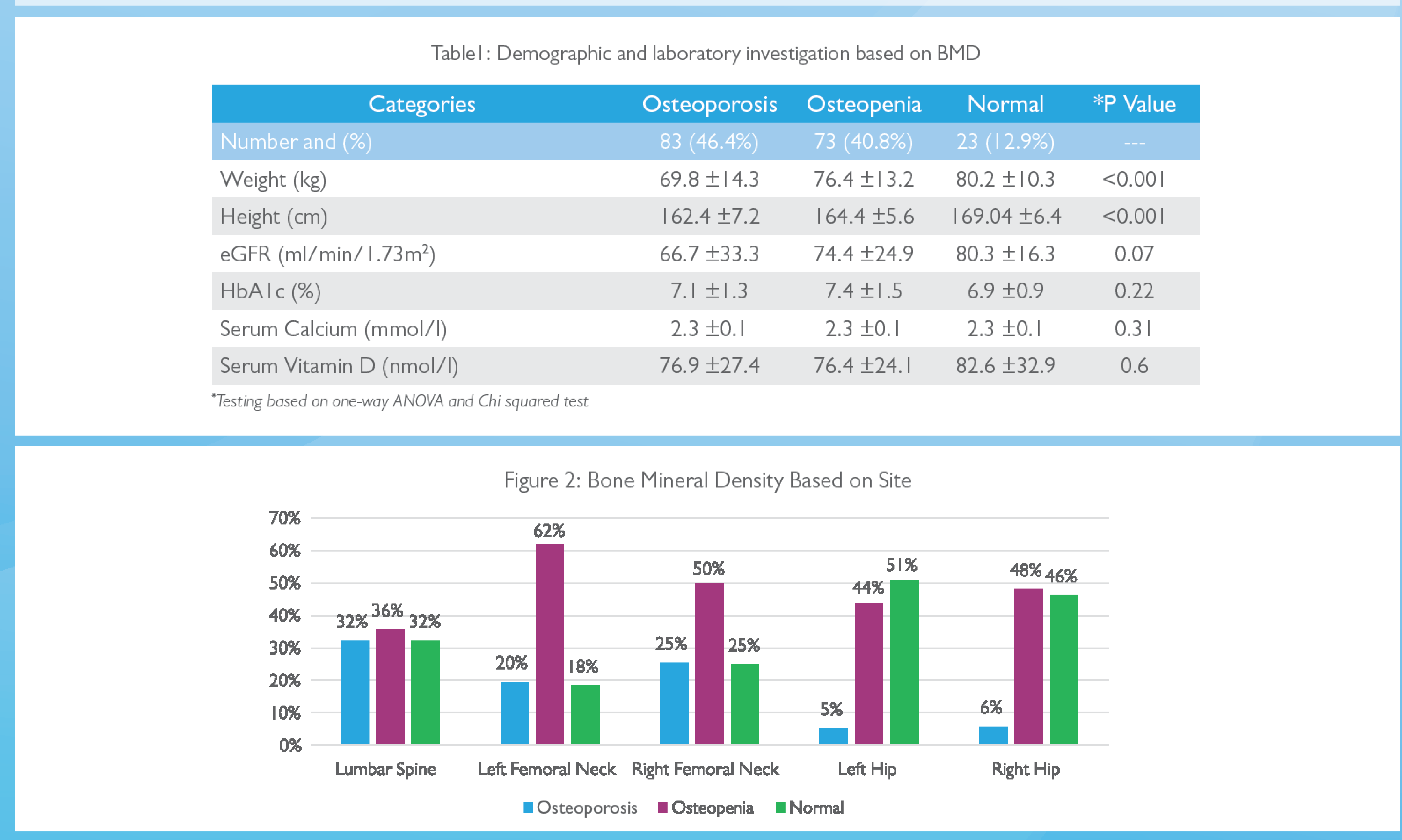

\section{Discussion}

- Compared to a previous pilot study on 45 Arab elderly men in UAE (3), our study is showing a higher percentage of osteoporosis in elderly Emirati men with diabetes.

- Local factors such as relative inactivity in old age and lack of sun exposure, may be partially responsible for high prevalence figures reported in this study. Diabetes may be an independent risk factor for osteoporosis in old Emirati men and merits further investigation in a larger group of patients.

- The possible explanation for the association between osteoporosis and height is that osteoporotic patients could have vertebral compression and thus reduced height.

- The high osteoporosis range T-scores concordance between femoral necks and hips suggests that DEXA at one site alone may be adequate for osteoporosis screening in the Emirati population.

\section{Selected References}

Al Attia H, et al. , Clin Rheumatol 2007; (26) | |23-| | 26.

2. Fawzy T, et al. , Journal of Osteoprosis 201 I; vol. 201 I, Article ID 876309, 4

3. Al Attia H, et al. , Rheumatol Int 2010 ; (30) 365-367.

\section{Acknowledgements}

Authors are grateful to ICLDC, its staff and patients for their help with this study. 